\title{
Miteinander Wissen schaffen. Partizipative Aktionsforschung als geographische Bildungspraxis zwischen Gesellschaft, Schule und Hochschule
}

\author{
*fabian.pettig@uni-graz.at, Institut für Geographie und Raumforschung, Universität Graz ( $\square$ korresp. Autor) \\ **info@orangotango.info, orangotango - Kollektiv für kritische Bildung und kreativen Protest \\ ${ }^{* * *}$ martina.neuburger@uni-hamburg.de, Institut für Geographie, Universität Hamburg
}

eingereicht am: 02.05.2021, akzeptiert am: 17.10.2021

Im Unterschied zu angelsächsischen und lateinamerikanischen Debatten findet die partizipative Aktionsforschung (PAF) in der Humangeographie im deutschsprachigen Raum bisher nur wenig Beachtung. Dabei adressiert PAF mehrere Kernanliegen transformativer Lern- und Forschungskontexte und verspricht daher, wertvolle Impulse in diesen Bereichen zu setzen. Im Beitrag werden diese Potenziale ausgelotet und PAF als kritisch-pädagogischer Denkrahmen für die geographische Bildungspraxis entfaltet. Das Vorgehen mündet in die Formulierung eines partizipativen Photovoice-Projekts zur Erforschung urbaner (Nicht-)Nachhaltigkeit mit Kindern und Jugendlichen in der Schule.

Keywords: Kritische Geographie, (Nicht-)Nachhaltigkeit, Photovoice, Transformatives Lernen, Emanzipation

\section{Creating knowledge together. Participatory action research as geographical educational practice between society, school, and university}

In contrast to Anglo-Saxon and Latin American debates, participatory action research (PAF) has so far received little attention in human geography in the German-speaking debate. Yet PAF addresses several core concerns of transformative learning and research contexts and therefore promises to provide valuable impulses in these areas. The paper explores these potentials and unfolds PAF as a critical pedagogical framework for educational practice in geography. This approach leads to the conceptualization of a photovoice project for participatory action research on urban (non)sustainability with children and adolescents in school.

Keywords: critical geography, (non)sustainability, photovoice, transformative learning, emancipation

\section{$1 \quad$ Einleitung}

Partizipative Aktionsforschung (PAF) ist mehr als eine (humangeographische) Forschungsmethode (vgl. Kindon \& Elwood 2009). Als spezifische Forschungspraxis vereint sie Forschung, politische Haltung, Bildung und Aktionen in einem Prozess, um theoretische und praktische Antworten auf gesellschaftliche Probleme zu finden. Sie versucht, die Kluft zwischen Wissenschaft und Gesellschaft zu überwinden, indem sie die Vermittlung zwischen den Erkenntnispositionen von Forschenden und Beforschten anstrebt und Alltags- sowie Praxiswissen mit akademischem Wissen in einen Dialog bringt. Diese Art der partizipativen und aktionsorientierten Forschung zeichnet sich aus durch einen hohen Grad an Engagement, kollektiver Vorgehensweise und kontextspezifischem Arbeiten. Seit den richtungsweisenden Texten von Lewin (1946) und Fals Borda (1978) wurde PAF in den Geisteswissenschaften breit praktiziert und unter einer Vielzahl an Begriffen rezipiert ${ }^{1}$. Es wird deutlich, dass sich diese

Im englischsprachigen Raum ist u. a. von action science; participatory action research; participatory rural appraisal; participatory learning and action; community-based participatory research; scholar activism; advocacy activist; militant research und practitioner research die Rede (Herr \& Anderson 2015: 18; Kindon et al. 2007: 12; Chatterton et al. 2010; Chambers 1994). Im lateinamerikanischen Kontext wird PAF z.B. unter pesquisa-açäo und 
Forschungspraxis in verschiedenen Ansätzen ausgestaltet. Dies ist auch in der Humangeographie der Fall, in der die aktions- und solidaritätsorientierten Arbeiten der radical geography seit den 1960er-Jahren besonderen Einfluss auf die Entwicklung politisch motivierter Formen von PAF nahmen (vgl. Katz 1996). An diese Arbeiten und unsere eigenen Kontexte anknüpfend, versucht der Beitrag die Brücke zwischen politischem Einsatz und (Hoch)Schule zu schlagen. Er ist inspiriert davon, dass PAF nach einer Zeit der Akademisierung und Professionalisierung in der (angelsächsischen) Humangeographie seit Ende der 1990erJahre wieder vermehrt als Lösungsansatz für soziale Probleme und Transformation in verschiedenen Formen und Räumen debattiert und ausprobiert wird (vgl. Castree 2000). Aktuellere Arbeiten betrachten die Teilnehmer*innen nicht mehr als Objekte, sondern als Subjekte im Forschungsprozess, die in Beteiligungsformaten eingebunden werden (vgl. Pain \& Francis 2003). Mit dem Ziel Kinder und junge Menschen in allen Phasen der Forschung einzubinden und damit ihre Perspektive stärker in den Mittelpunkt zu rücken, liefern insbesondere die (critical) geographies of children and young people kreative Impulse. Aufbauend auf methodischen Innovationen aus den cultural studies werden hier Widersprüche und Herausforderungen bei der Umsetzung von PAF benannt und Lösungsvorschläge erarbeitet (vgl. Evans et al. 2017). In PAF-Formaten werden darüber hinaus politischer Aktivismus und kritische Forschung bewusst zusammengeführt (vgl. Routledge 2017; Scheidel et al. 2020).

Auch in der deutschsprachigen Humangeographie werden universitäre und alltägliche Räume durch die Verbindung von kritischer Forschung, sozial-gültiger - d.h. sowohl für die Lebenswirklichkeiten der forschenden Akteur*innen als auch für die Gesellschaft insgesamt relevanter - Anwendung und transformativer Bildung ${ }^{2}$ miteinander verknüpft (vgl. Kuge et al. 2020). Das Panorama reicht von aktivistischer Lobbyarbeit für nachhaltige Wohnformen und ein Recht auf Stadt (vgl. Mießner 2020) sowie geographischem

investigación militante diskutiert (vgl. Bartholl 2015). Ferner gibt es disziplinspezifische Ausrichtungen wie activist geography (vgl. Routledge 2009), militant cartography (vgl. Counter Cartographies Collective, Mason-Deese \& Dalton 2012) oder teacher research (vgl. Cochran-Smith \& Lytle 1993). In der deutschsprachigen Diskussion können unterschiedliche Strömungen wie die Handlungsforschung, Tatforschung, aktivierende Sozialforschung und politisierende Sozialforschung als Unterkategorien der PAF verstanden werden (Halder 2018: 45, 66).

2 Transformative Bildung wird hier im Sinne von Paulo Freires (1971) educação popular verstanden, eine dialogische Herangehensweise, in der alle Beteiligten alltägliche Situationen hinsichtlich sozio-kultureller Hierarchien problematisieren, gemeinsame Widersprüche in ihnen gemeinsam (sprachlich) begreifen und diese dadurch verändern lernen.
Aktivismus in urbanen Gärten (vgl. Halder 2018) über transformative Perspektiven in kritisch-geographischer Lehre (vgl. Domann \& Liebscher 2020) bis hin zu Ansätzen partizipativen Monitorings als Instrument zur Umsetzung von Good Local Governance (vgl. Segebart 2007) und auto-ethnographischen Analysen historisch kolonialer Einschreibungen in Formen des Geographie-Machens (vgl. Singer 2019).

Vor diesem vielgestaltigen Hintergrund von PAF verfolgt der vorliegende Beitrag das Ziel, deren Grenzverläufe in unterschiedlichen Verwendungszusammenhängen auszuloten und Einsatzmöglichkeiten zu diskutieren. Hierzu werden die Umsetzung von PAFProjekten dargelegt, eine universitäre Lehrveranstaltung skizziert und ein Unterrichtsbaustein formuliert. Der Beitrag schließt mit einer dialogischen Reflexion des Ansatzes aus fachwissenschaftlicher und fachdidaktischer Sicht.

\section{Prüfsteine Partizipativer Aktionsforschung: Zirkularität, Beteiligung, Gütekriterien}

Durchführungen von PAF sind stärker durch ihre Grundprinzipien als ihre methodischen Vorgehensweisen vereint. Die Auswahl empirischer Methoden variiert stark in Abhängigkeit vom Forschungs- und Aktionsziel. Orientierung bei der Durchführung bieten die sich wiederholenden Phasen der Analyse, Planung, Aktion und Reflexion (siehe Abb. 1), das Kontinuum der Positionalitäten mit verschieden starken Graden an Identifikation mit dem Forschungskontext (Herr \& Anderson 2015: 45 ff.) und die Auswahl innovativer, qualitativer und partizipativer Forschungsmethoden (Halder 2018: 341) ${ }^{3}$. Ein kritisches Bewusstsein für die Stufen der Partizipation, die sich im Bild der „Partizipationsleiter" ${ }^{4}$ (Arnstein 1969) widerspiegeln, markiert die Grenze zu einer pseudo-partizipativen Forschung, die unter emanzipatorischem Deckmantel die Mitwirkenden instrumentalisiert (siehe Abb. 2).

PAF versucht objektiv, aber nicht neutral zu sein (Sousa Santos 2018: 261). Das bedeutet, dass

\footnotetext{
Weitere aktuelle Methoden der PAF sind u. a. die forschende Lehre im Urban Transition Lab (vgl. Domann \& Liebscher 2020) und kritische Kartierungen (vgl. Dammann \& Michel i. E.).

4 Arnsteins Partizipationsleiter wurde auch für die gemeinsame Forschung mit Kindern und Jugendlichen adaptiert (vgl. Hart 1992); hier reicht diese von der manipulativen Vereinnahmung bis hin zu durch Kinder und Jugendliche selbst initiierter, durchgeführter und reflektierter Forschung, in denen Erwachsene lediglich unterstützend wirken. Hart (2008) betont, dass sein Leiter-Modell keine Hierarchie oder gar unmittelbare Handlungsempfehlungen impliziert; vielmehr kommt es immer auf die konkreten Kontexte an, in denen sich Mitwirkung auf unterschiedliche Weise realisieren kann.
} 


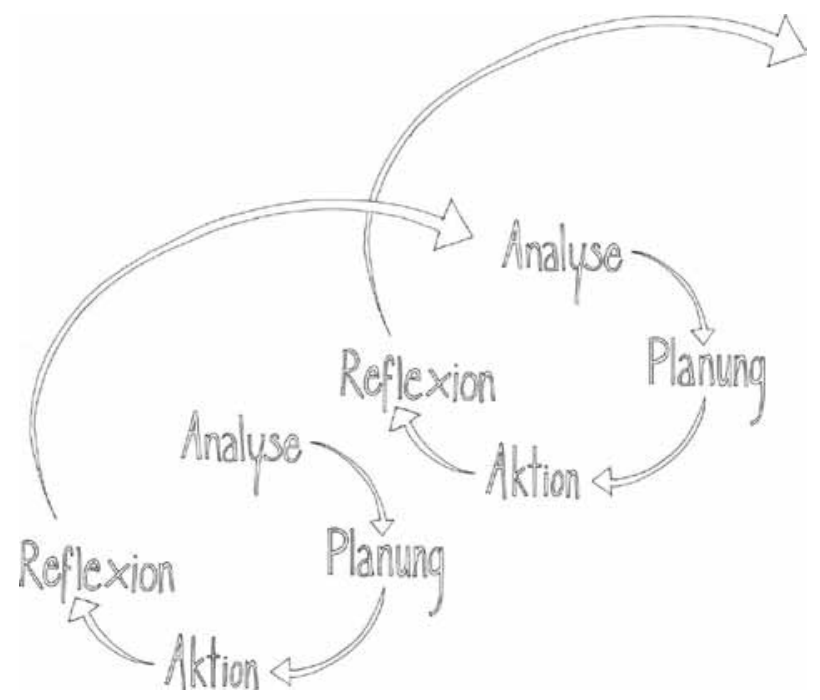

Abb. 1: Zyklischer Ablauf partizipativer Aktionsforschung (angelehnt an Halder 2018: 77).

eine klare Positionierung der Forschenden und die gleichzeitige Einhaltung wissenschaftlicher Qualitätsstandards keinen Widerspruch darstellen. Um sicherzustellen, dass der Spagat zwischen politischer Positionierung und akademischer Wissensproduktion gemeistert wird, sollten die PAF-spezifischen Qualitätskriterien beachtet werden (Halder 2018: 75). Eine Gültigkeit der Ergebnisse ist dann gewährleistet, wenn sowohl der theoretische als auch der aktionsbezogene Teil der Arbeit einen Beitrag zur Lösung des gesellschaftlichen Problems leisten kann, das den Ausgangspunkt für die Arbeit darstellt (Herr \& Anderson 2015: 67). Transformative Gültigkeit lässt sich an dem Grad messen, inwieweit die beteiligten Akteur*innen kritische Lernprozesse in Bezug auf bestehende gesellschaftliche Verhältnisse durchlaufen und dadurch zu eigenen gesellschaftsrelevanten Inter- ventionen befähigt werden (ebd.: 69). Die kollaborative Gültigkeit prüft, inwieweit die Forschung einen lokalen Anwendungsbezug entfaltet, der auf den Bedürfnissen der beteiligten Akteur*innen beruht und Maßstäben der sozialen Gerechtigkeit unterliegt (ebd.). Da Aktionsforschende häufig Doppelrollen einnehmen und verschiedene Erkenntnispositionen sowie Wissensformen zusammenzubringen, besteht das Risiko, wissenschaftliche Validität durch eine zu große Identifikation mit dem Feld zu verringern (ebd.: 73). Deshalb empfiehlt sich die Einhaltung der Gütekriterien qualitativer Forschung wie Transparenz, Gegenstandsangemessenheit, empirische Verankerung, Relevanz, Kohärenz und reflektierte Subjektivität zur Reflexion der eigenen Praxis (vgl. Steinke 2010). Um einen fortwährenden und sich vertiefenden Lern- und Reflexionsprozess bei allen Beteiligten und so die Gültigkeit des Prozesses zu gewährleisten, eignen sich Verfahren mit Triangulation, die eine Kombination verschiedener Methoden und die Integration verschiedener Perspektiven vorsehen (Herr \& Anderson 2015: 68).

\section{Partizipative Aktionsforschung in der geographischen Lehre - Erfahrungen aus Hochschulseminaren}

Die oben dargestellten Gütekriterien verweisen auf den transformativen Anspruch von PAF (Halder 2018: 76). In der Debatte über eine praxisbezogene Rückbesinnung und theoretisch fundierte Neuausrichtung der kritischen Humangeographie mit genau diesem Anspruch werden auch die Hörsäle und Seminarräume zu Orten sozialer Auseinandersetzungen (vgl. Castree 2000). Im angelsächsischen Dis-

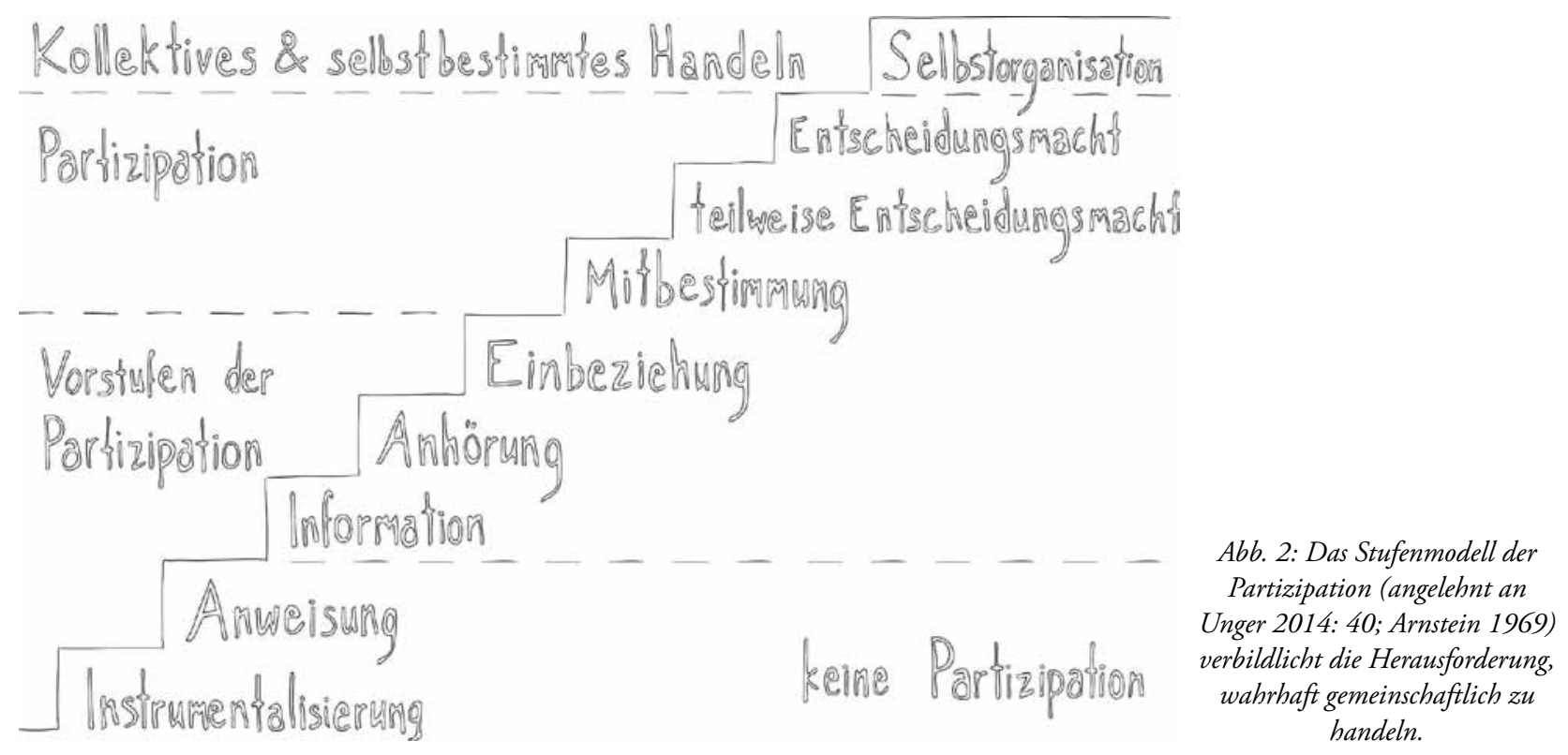


Tab. 1: PAF-Phasen des Geländepraktikums und deren Elemente (eig. Darstellung)

\begin{tabular}{|c|c|c|c|c|}
\hline $\begin{array}{c}\text { Vortreffen \& 1. Block } \\
\text { (Analyse) }\end{array}$ & $\begin{array}{c}\text { 2. Block } \\
\text { (Analyse \& Planung) }\end{array}$ & $\begin{array}{l}\text { Geländepraktikum } \\
\text { (Planung vor Ort) }\end{array}$ & $\begin{array}{l}\text { Geländepraktikum } \\
\text { (Aktionen vor Ort) }\end{array}$ & $\begin{array}{l}\text { Forschungsbericht } \\
\text { (Reflexion) }\end{array}$ \\
\hline $\begin{array}{l}\text { Gruppenbildung und } \\
\text { thematische Lektürearbeit }\end{array}$ & $\begin{array}{l}\text { Vorbereitung: } \\
\text { Forschungsinteressen } \\
\text { konkretisieren }\end{array}$ & Ankommen vor Ort & $\begin{array}{l}\text { Beginn der Datenerhe- } \\
\text { bungen, Schreiben von } \\
\text { Feldtagebüchern und } \\
\text { Umsetzung geplanter } \\
\text { Aktionen }\end{array}$ & $\begin{array}{c}\text { Reflexion der gemachten } \\
\text { Erfahrungen in Abgleich } \\
\text { mit Theorie }\end{array}$ \\
\hline $\begin{array}{c}\text { Einarbeitung in den } \\
\text { lokalen Kontext Neapels } \\
\text { und spezifische Themen- } \\
\text { felder }\end{array}$ & $\begin{array}{l}\text { PAF-Methodentraining } \\
\text { und -diskussion mit Fo- } \\
\text { kus auf Umsetzung und } \\
\text { Reflexion der Forschung }\end{array}$ & $\begin{array}{l}\text { Stadtexkursionen mit } \\
\text { lokalen Akteur*innen } \\
\text { und erster Austausch im } \\
\text { Forschungskontext }\end{array}$ & $\begin{array}{c}\text { Produktion nicht-aka- } \\
\text { demischer Ergebnisse } \\
\text { (audio-visuelles Material } \\
\text { od. öffentliche Kulturver- } \\
\text { anstaltung) }\end{array}$ & $\begin{array}{c}\text { Forschungsbericht fina- } \\
\text { lisieren }\end{array}$ \\
\hline $\begin{array}{l}\text { Zugänge zu möglichen } \\
\text { Forschungskontexten } \\
\text { (z. B. Kulturzentren, } \\
\text { Gemeinschaftsgärten) } \\
\text { aufbauend auf bestehen- } \\
\text { den Kontakten }\end{array}$ & $\begin{array}{l}\text { Entwurf von For- } \\
\text { schungsskizzen und } \\
\text { Kontaktaufnahme der } \\
\text { Gruppen mit lokalen } \\
\text { Ansprechpartner*innen }\end{array}$ & $\begin{array}{l}\text { Partizipative Planung re- } \\
\text { levanter Fragestellungen } \\
\text { und konkreter Aktionen } \\
\text { mit lokalen Akteur*innen }\end{array}$ & $\begin{array}{l}\text { Dokumentation und evtl. } \\
\text { erneute Zyklen }\end{array}$ & $\begin{array}{c}\text { Weiterführender } \\
\text { Austausch mit lokalen } \\
\text { Akteur*innen }\end{array}$ \\
\hline
\end{tabular}

kurs sprachen Kindon und Elwood (2009: 20) im Bereich der geographischen Hochschullehre bereits vor einigen Jahren von einem "participatory turn“. Im deutschsprachigen Kontext wird PAF in kritischgeographischer Lehre u. a. als Mittel für die Thematisierung gesellschaftlicher Aushandlungsprozesse, Widersprüche und Konflikte erprobt (vgl. Domann \& Liebscher 2020).

Auch die Forschungs- und Bildungspraxis von kollektiv orangotango ${ }^{5}$ bezieht sich stark auf PAF-Ansätze. Das Kollektiv versucht in einer, geographischen Bildung von unten' Kritik an Machtverhältnissen, Transformation und Bildungsprozesse in und außerhalb der Universität sowie in den Zwischenräumen zusammenzubringen (Halder \& Schweizer 2020: 258). In unterschiedlichen universitären Lehrveranstaltungen an der Universität Hamburg und der FU Berlin, an denen kollektiv orangotango in den letzten Jahren beteiligt war, wurden PAF-Ansätze thematisiert und erprobt. Allerdings bieten einsemestrige Lehrveranstaltungen kaum den zeitlichen Rahmen, der für die Einhaltung der oben definierten Gütekriterien nötig wäre. Zur Erarbeitung eigener Methoden und Standards als Rahmen für mögliche Anwendungen von PAF in studentischen Forschungsprojekten, bedarf es weiterer Diskussion sowie der Systematisierung bereits gemachter Erfahrungen. Diesen Text verstehen wir als Beitrag hierzu.

In dem von einer Professorin für Humangeographie und einem Mitglied des Kollektivs geleiteten Geländepraktikum Peripherien in Bewegung

\footnotetext{
Das kollektiv orangotango entsteht seit 2008 im freundschaftlichen Umfeld kritischer Geograph*innen die sich mit kritischer Bildungsarbeit, selbstorganisierten Strukturen und künstlerischen Interventionen, die zur Reflexion und Veränderung der bestehenden Verhältnisse beitragen, beschäftigen (siehe orangotango.info).
}

- Prekarisierung und Widerstand in der Metropolregion Neapel im Wintersemester 2019/20 an der Universität Hamburg wurden Methoden der PAF gemeinsam mit Studierenden erprobt. Ausgangspunkt der zweiwöchigen Lehrveranstaltung war die Annahme, dass sich im Kontext krisenhafter Wirtschaft und autoritärer Politik Prekarisierung und Unterdrückung, aber auch Selbstorganisation und widerständige Praktiken, in städtischen Räumen konzentrieren (vgl. Zibechi 2011). Durch den Austausch mit unterschiedlichen Aktivist*innen sollten die Studierenden untersuchen, wie Prekarisierungsund Unterdrückungsformen begegnet, Freiraum geschaffen und gemeinschaftliche Stadtgestaltung in Neapel organisiert wird. Das Geländepraktikum gliederte sich in vier Phasen, die sich am Zyklus der Aktionsforschung orientierten (siehe Tab. 1). Auch wenn formal das Schreiben eines Forschungsberichts als zu benotendes Ergebnis im Vordergrund stand, wurden die Studierenden bereits in der Analysephase ermutigt, sich darüber hinaus vor Ort einzubringen, den Sinn und Zweck ihrer Forschung mit lokalen Akteur*innen zu diskutieren sowie Ergebnisse und Materialien zu produzieren, die in den Forschungskontext zurückgetragen werden können.

Diese Ansprüche der PAF erforderten von den Studierenden in der Planungs- und Aktionsphase vor Ort ein hohes Maß an Engagement, Flexibilität und Offenheit. Da nur wenige der 19 Studierenden Italienisch sprachen, waren sie zwangsläufig auch darauf angewiesen, mit non-verbalen Methoden der Datenerhebung und -auswertung zu arbeiten.

Trotz der großen Herausforderungen konnten jene Gruppen, die sich für einen an PAF orientierten Ansatz entschieden, sowohl die Forschung in Neapel als auch die Erarbeitung der Ergebnisse überwiegend 


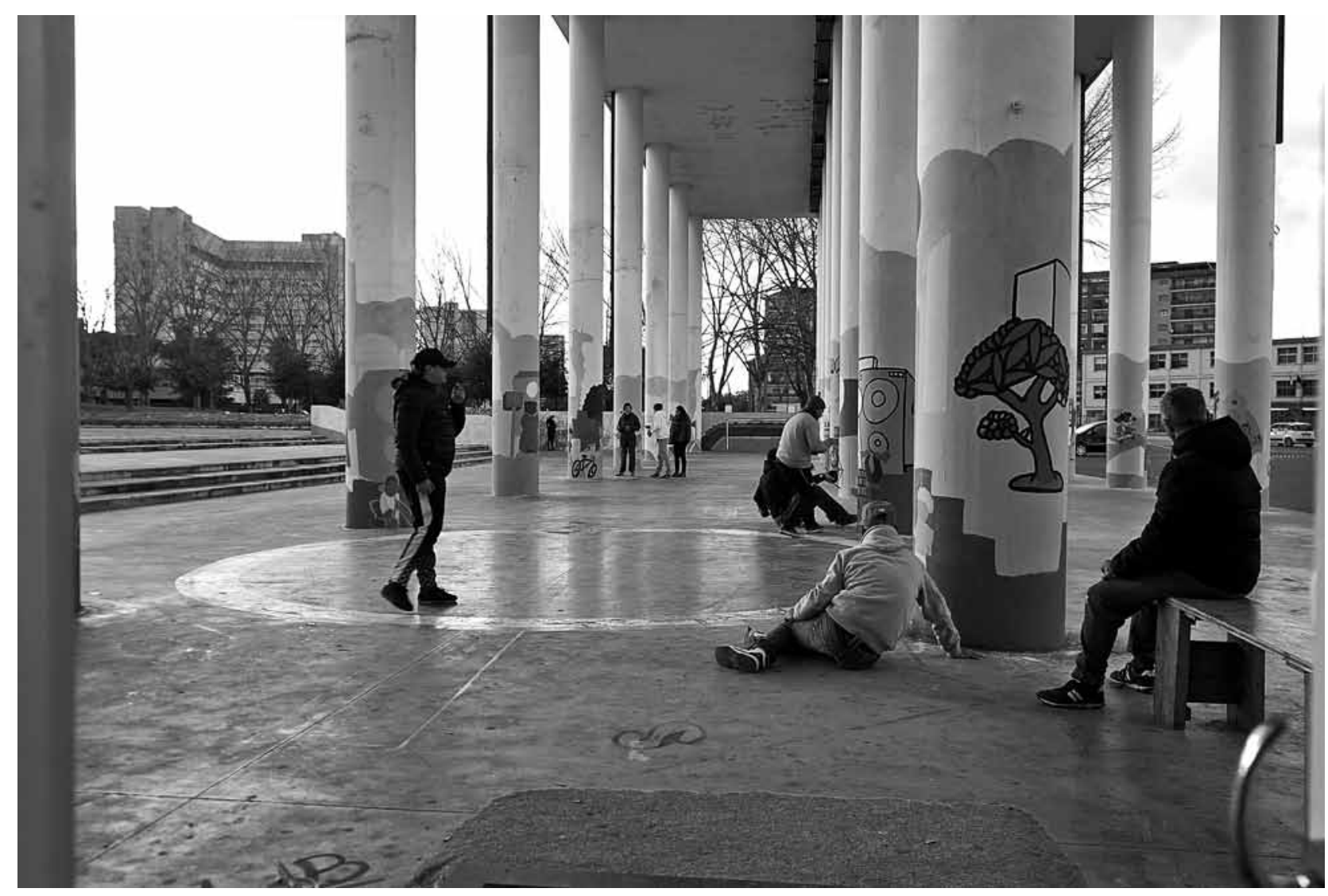

Abb. 3: Kollektive Wandbemalung auf der zwischen extrem rechten Mobilisierungen, informeller Ökonomie und kritisch-pädagogischer Basisarbeit umkämpften Piazza Ciro Esposito. Die Gruppe, die an der Wandbemalung aktiv und forschend beteiligt war, produzierte parallel ein kurzes Video des Entstehungsprozesses, welches Teil einer Crowdfunding-Kampagne der lokalen NGO wurde. ${ }^{6}$ (Foto: Meincke 2020)

im Sinne der PAF durchführen. So brachten sich verschiedene Gruppen intensiv in die laufenden Aktivitäten der untersuchten Kontexte ein und trugen einzelne Aktionen maßgeblich mit. Dabei beteiligten sie sich tatkräftig an Arbeiten, die unabhängig von ihrer Forschung zu diesem Zeitpunkt anfielen oder erörterten mit den Partner*innen, welchen praktischen Beitrag sie im Rahmen ihrer Forschungsarbeiten leisten könnten. Eine Gruppe untersuchte die „emotional geography“ (vgl. Davidson \& Milligan 2004) sowie kollektive Wissensproduktionen in selbstverwalteten Theater- und Zirkus-Kursen mithilfe einer auto-ethnographischen Studie. Eine weitere Gruppe legte eine intersektionale Perspektive an, um safe spaces innerhalb eines Centro Sociale mithilfe der intersektional orientierten Darstellungsform Relief Maps zu untersuchen (vgl. Rodó-de-Zárate 2014) und organisierte Diskussionsveranstaltungen mit kulinarischem und filmischem Begleitprogramm. Ein weiteres Projekt widmete sich künstlerischen Interventionen zur Aneignung des öffentlichen Raums am Beispiel einer kollektiven Wandbemalung (siehe Abb. 3).

Video I Viaggi del Mammuttino: https://vimeo.com/441755941
In der letzten Phase verfassten die Studierenden Forschungsberichte und reflektierten im Zuge dessen die gemachten Erfahrungen. Obwohl bei der Begutachtung der Forschungsberichte deutlich wurde, dass nicht alle Gruppen sämtliche Gütekriterien von PAF erreichen konnten, gab es nur einzelne Studierende, die sich kaum persönlich mit der Fragestellung identifizierten oder einen intensiven Austausch mit unterschiedlichen Akteur*innen eingingen. Trotz der geringen zeitlichen Ressourcen gelang es einigen Gruppen außerdem, neben dem obligatorischen Forschungsbericht, weitere für den beforschten Kontext nutzbare Materialien zu generieren.

\section{PAF als Framework zur Ermöglichung transformativer Lernprozesse im Geogra- phie- und GW-Unterricht}

Formate der Aktionsforschung werden inzwischen als eines der "Schwerpunktegebiete geographiedidaktischer Forschung" (Rinschede \& Siegmund 2020: 34) verstanden. Wenngleich die Umsetzung entsprechend vielfältig ist, wird Aktionsforschung in der Geographiedidaktik häufig vor allem in Anlehnung an die Über- 
legungen von Altrichter et al. (2018) zur Entwicklung von Unterricht und Lehr-Lern-Umgebungen, bspw. durch Lehrkräfte (z. B. Kanwischer \& Rhode-Jüchtern 2002), durch Hochschullehrende (z. B. Schulze et al. 2018), aber auch gemeinschaftlich durch Lehrkräfte und universitär Forschende (z. B. Leder 2015) eingesetzt. Explizite Verweise auf die PAF finden sich in der geographiedidaktischen Diskussion hingegen eher vereinzelt (u.a. Pichler et al. 2021). ${ }^{7}$ Dies mag sicherlich auch mit der Vielzahl an partizipativen Ansätzen ${ }^{8}$ zusammenhängen, deren Anliegen und Verfahren sich teilweise überschneiden, sodass die Übergänge bisweilen fließend sind (Bergold \& Thomas 2012; Resch \& Knapp 2020). Partizipative Ansätze und Methoden, in denen Kinder und Jugendliche eine bedeutende Rolle in geographischen Forschungsprozessen einnehmen, sind in der Geographiedidaktik gut etabliert, bspw. intermediale Mapping-Verfahren (vgl. Pettig 2019), reflexive Fotografie (vgl. Eberth \& Röll 2021) oder SpatialCitizenship-Education (vgl. Pokraka \& Gryl 2018). In diesen wird häufig auch ein weiteres Kernanliegen von PAF verfolgt: Perspektiven von im öffentlichen Diskurs marginalisierten Gruppen sichtbar zu machen, um Partizipation zu ermöglichen und Mündigkeit zu fördern.

Im Rahmen dieses Beitrags wird PAF im Geographieunterricht als Framework für gemeinschaftliche Forschungsprozesse von Schüler*innen und Lehrer*innen (sowie ggf. weiteren Akteur*innen) verstanden. Dabei organisieren Lehrkräfte die Rahmenbedingungen und treten als Dialogpartner*innen im Prozess auf, sodass Schüler*innen soweit als möglich an allen Phasen der Forschungsvorhaben umfassend partizipieren können. Schüler*innen nehmen also die Rolle von „Co-Forscher*innen“ (vgl. Unger 2014) ein, die eigene Fragestellungen innerhalb eines thematischen Horizonts verfolgen können. PAF in den Geographie- und GW-Unterricht zu implementieren lässt sich als Balanceakt zwischen selbstbestimmter partizipativer Forschung einerseits und der Orientierung an institutionellen Rahmenbedingungen sowie fachspezifischen Bildungsanliegen andererseits bestimmen. Das Unterrichtsbeispiel will zwischen diesen beiden Perspektiven sinnstiftend vermitteln; der Grad der Partizipation bewegt sich dabei zwischen Einbeziehung und Mitbestimmung.

Im Rahmen dieses Beitrags können die Gemeinsamkeiten, Unterschiede und Verflechtungen der schillernden Rezeptionsgeschichte der Action Research im internationalen bzw. auch deutschsprachigen Raum in ihren jeweiligen Eigenheiten nicht näher beleuchtet werden. Unger (2014) bietet einen guten Einblick in die einzelnen Diskurslinien.

8 Neben participatory rural appraisal, community-based participatory research, scholar activism, transition research oder citizen science bspw. auch das in der Geographiedidaktik zunehmend Beachtung findende Service Learning oder auch Projektbasiertes Lernen und Forschendes Lernen.
Die unterrichtliche Umsetzung von PAF wird in diesem Beitrag durch zwei Schwerpunktsetzungen geleitet: 1. Zum einen dient die partizipative Forschungsmethode „Photovoice“ (vgl. Wang \& Burris 1994, 1997) der methodischen Konkretisierung. Die Methode ist unkompliziert mit Kindern und Jugendlichen einzusetzen und gilt als besonders geeignet, Veränderungsprozesse auf individueller und gemeinschaftlicher Ebene anzustoßen und zu unterstützen (Wihofszky et al. 2020: 87). Photovoice will Co-Forscher*innen eine, fotografische Stimme' geben, indem diese mit der Kamera Daten zu einer Forschungsfrage erheben, die dann gemeinsam analysiert und reflektiert werden, um die Erkenntnisse einem Zielpublikum zu präsentieren.

2. Zum anderen wird der thematische Schwerpunkt auf Forschungsfragen zu Nachhaltigkeit und nachhaltiger Entwicklung im Alltag und Umfeld der Schüler*innen gelegt. Denn da ein ausgewiesenes Ziel von PAF in der Demokratisierung von Forschung und Wissensproduktion liegt, lässt sich hierüber auch ein Anliegen einer emanzipatorischen Bildung für nachhaltige Entwicklung (BNE) adressieren. Im Gegensatz zu instrumentellen Ansätzen, die darauf abzielen, Expert*innenwissen zu vermitteln und ein bestimmtes - als, nachhaltig' verstandenes - Verhalten zu fördern, fokussiert eine emanzipatorische Idee einer BNE die kritisch-reflexive Auseinandersetzung mit den Widersprüchen und Herausforderungen von Nachhaltigkeit und nachhaltiger Entwicklung (u. a. Vare \& Scott 2007).

Mit Blick auf partizipative Forschungsprojekte mit konkretem Mensch-Umwelt-Bezug konstatieren Mordock und Krasny (2001), dass diese kritisches Denken und die Fähigkeit zur Problemlösung fördern, das Verständnis für wissenschaftliches Denken und Vorgehen stärken, über Selbstwirksamkeitserfahrungen und Bewusstseinsbildung zum Empowerment der Beteiligten beitragen und als Motor sozialer Transformationsprozesse und ökologischen Wandels fungieren.

PAF-Formate besitzen also sowohl das Potenzial, den in der Nachhaltigkeitsdebatte häufig diskutierten Knowledge-Action-Gap, also die Kluft zwischen Wissen und Handeln, zu adressieren (vgl. Kerkhoff \& Lebel 2006), als auch, sich kritisch und reflexiv ins Verhältnis zu hegemonialen Nachhaltigkeitsdiskursen zu setzen. Damit stehen Selbstwirksamkeitserfahrungen und die dialogische Aushandlung von Haltungen angesichts nachhaltigkeitsbezogener Problemstellungen im Vordergrund, um die Co-Forscher*innen dabei zu unterstützen, aktiv an der Gestaltung einer zukunftsfähigen und sozial gerechten Welt teilhaben zu können. In diesem Sinne erscheint PAF grundsätzlich 
- und Photovoice hier im Speziellen - überaus geeignet, „transformatives Lernen“ (vgl. Singer-Brodowski 2016; Pettig 2021) zu ermöglichen.

\section{5 (Nicht-)Nachhaltigkeit mit Photovoice sichtbar machen - ein Unterrichtsbeispiel}

Zielgruppe und Vorwissen. Das Unterrichtsbeispiel eignet sich für alle Schulformen ab der Klassenstufe 10, da für die Feldarbeit auch das Schulgelände verlassen werden muss. Differenzierungsmöglichkeiten bieten sich in der Stringenz der methodischen Anleitung (offen oder entlang von Protokollbögen) sowie in den wiederkehrenden Dialog- und Reflexionsphasen. Es ist sinnvoll, dass die Schüler*innen in Vorbereitung auf das Projekt bereits mit Grundlagen der Klimakrise und Nachhaltigkeitsdebatte vertraut sind (anthropogene Einflüsse, gängige Strategien des (urbanen) Klimaschutzes, etablierte Konzepte wie Nachhaltigkeitsdreieck bzw. -viereck und Vorrangmodell). Dieses Vorwissen wird in Dialog- und Reflexionsphasen aufgegriffen, erfahrungsbezogen angereichert und sollte auch hinsichtlich deren eingeschriebenen Logiken an konkreten Erfahrungen aus der Forschungspraxis problematisiert werden.

Thematischer Rabmen. Der Unterrichtsbaustein problematisiert (nicht-)nachhaltige Situationen, Strukturen und Prozesse im Alltag und dient dem Suchen nach und der kritischen Diskussion von Möglichkeiten, auf diese Verhältnisse aufmerksam zu machen und Veränderungsprozesse anzuregen. Im Zentrum steht die sachlich angemessene Erarbeitung und Diskussion der fachlichen und politischen Gründe für die jeweiligen nicht-nachhaltigen Entwicklungen, um hierüber Perspektiven für das eigene und kollektive Handeln zu gewinnen (vgl. Euler 2014). ${ }^{9}$ Damit oszillieren die einzelnen Forschungsvorhaben der Schüler*innen innerhalb des Bausteins zwischen einer individuellen und einer gesellschaftlich-strukturellen Ebene von

\footnotetext{
Es bietet sich daher auch an, das Projekt in Kooperation mit weiteren Akteur*innen oder Expert*innen zu realisieren, z. B. Kolleg*innen, Bildungsakteur*innen oder Praxispartner*innen diese können sowohl bei der Erarbeitung eines gemeinsamen Problemhorizonts, als auch in den wiederkehrenden Dialogphasen einbezogen werden, und wertvolle Impulse liefern. Auch wenn Kooperationen durchaus wünschenswert sind, erhöht sich der Vorbereitungs- und Organisationsaufwand hierdurch deutlich. Für ein gelingendes Photovoice-Projekt in der Schule sind solche Kollaborationen zwar überaus bereichernd, aber nicht zwingend notwendig.

10 Der Begriff, (Nicht-)Nachhaltigkeit' soll verdeutlichen, dass die Grenzverläufe der beiden zusammengefügten Begriffe in Zeiten inflationärer Verwendung des Nachhaltigkeitsbegriffs, global verzweigter Lieferketten und Green-Washing zusehends verschwimmen und häufig nicht abschließend geklärt sind.
}

(Nicht-)Nachhaltigkeit. ${ }^{10}$ Aufgabe der Lehrkraft ist es, die Widersprüche und Spannungen einer ,nachhaltigen Entwicklung' zwischen individueller Verantwortung und systemischen Bedingungen von (Nicht-) Nachhaltigkeit immer wieder ins Bewusstsein der CoForscher*innen zu rücken. Es geht nicht darum, mit den Schüler*innen einfache Lösungen zu erarbeiten und individuelle Pflichten für den Alltag zu formulieren, sondern eine „Einsicht in nicht-nachhaltige Prozesse [zu ermöglichen] und über die Reflexion der Ursachen ein Problembewusstsein [zu] eröffnen, welches vielleicht imstande ist, nachhaltige Auswege aus den globalen Krisen ins Auge zu fassen" (Kehren \& Winkler 2019: 377).

Bausteine zur Umsetzung in der Schule. Zur Realisierung von PAF-Projekten in der Schule sind mehrere Bausteine sinnvoll (siehe Tab. 2). Die tabellarische Übersicht dient lediglich als Anregung und sollte an die entsprechenden konkreten Schulkontexte angepasst werden. Mögliche Formate für PAF-Projekte im Unterricht können z. B. Projektunterricht, Nachmittags-AGs oder spezielle Modulkonzepte und offene Lernarrangements sein. Bei der Formulierung und Ausgestaltung der Bausteine haben wir uns an den Phasen von PAF und dem „Handlungsrahmen für transformatives Lernen" (vgl. Pettig 2021) orientiert. Das organisatorische Gerüst sowie die Hinweise zur Realisierung speisen sich gleichermaßen aus unseren Erfahrungen aus mehreren partizipativen Forschungsprojekten in der Bildungsarbeit mit Kindern und Jugendlichen im schulischen und außerschulischen Bereich, in denen wir unterschiedliche Methoden (u. a. kartographische, fotografische und künstlerische Verfahren) verwendeten, als auch einschlägiger Literatur zum Thema (vgl. Unger 2014; Wöhrer et al. 2017; Hartung et al. 2020).

Tab. 2: Bausteine eines Photovoice-Forschungsprojekts in der Schule

\begin{tabular}{|c|c|c|c|}
\hline $\begin{array}{c}\text { Baustein } 1 \\
\text { Analyse \& } \\
\text { Planung }\end{array}$ & $\begin{array}{c}\text { Baustein } 2 \\
\text { Aktion \& } \\
\text { Reflexion }\end{array}$ & $\begin{array}{l}\text { Baustein } 3 \\
\text { Kuratieren \& } \\
\text { Präsentieren }\end{array}$ & Follow-Up \\
\hline $\begin{array}{l}\text { Problemanalyse } \\
\text { (Nicht)Nach- } \\
\text { haltigkeit im } \\
\text { Alltag und } \\
\text { Stadtteil }\end{array}$ & \multirow[t]{2}{*}{$\begin{array}{l}\text { Datenerhe- } \\
\text { bung mit } \\
\text { Photovoice }\end{array}$} & $\begin{array}{l}\text { Forschungs- } \\
\text { vorhaben fi- } \\
\text { nalisieren und } \\
\text { kuratieren }\end{array}$ & \multirow{3}{*}{$\begin{array}{l}\text { ggf. Durchfüh- } \\
\text { rung weiter- } \\
\text { führender } \\
\text { Aktionen }\end{array}$} \\
\hline $\begin{array}{l}\text { Formulie- } \\
\text { rung von } \\
\text { Forschungs- } \\
\text { fragen }\end{array}$ & & $\begin{array}{c}\text { Präsentation } \\
\text { und Diskussion } \\
\text { der Ergebnisse } \\
\text { unter Ein- } \\
\text { bindung des } \\
\text { Zielpublikums }\end{array}$ & \\
\hline $\begin{array}{l}\text { Planung der } \\
\text { Vorhaben und } \\
\text { Feldphasen }\end{array}$ & $\begin{array}{l}\text { Dialog und } \\
\text { Reflexion }\end{array}$ & $\begin{array}{l}\text { Debriefing } \\
\text { Forschung } \\
\text { evaluieren und } \\
\text { Sichtweisen } \\
\text { aktualisieren }\end{array}$ & \\
\hline
\end{tabular}




\section{Baustein 1: Rahmenbedingungen klären und For- schungsvorhaben planen}

Probleme bestimmen, analysieren und Fragen formulieren. Eingangs wird der gemeinsame Horizont der Forschungsvorhaben erarbeitet und die übergeordnete Fragestellung des Projekts formuliert: Wo begegnet uns (Nicht-)Nachhaltigkeit in unserem Alltag und wie wird diese sicht- und erlebbar? Die Schüler*innen tauschen sich hierzu in Kleingruppen zu ihren Vorstellungen von (Nicht-)Nachhaltigkeit aus: Was bedeutet Nachhaltigkeit; was Nicht-Nachhaltigkeit? Welche konkreten Situationen, Handlungen, Gegenstände sind nachhaltig; welche nicht? Woran machen wir das fest? Die Überlegungen werden nach Themen geordnet und in Begriffswolken visualisiert, um geeignete Kriterien zur Bewertung einzelner Sachverhalte über Mehrfachnennung abzuleiten. Ziel dieses Auftakts ist es, das Vorwissen der Schüler*innen zu aktivieren, verschiedene Vorstellungen von (Nicht-)Nachhaltigkeit sichtbar zu machen und thematische Anknüpfungspunkte für die Forschungsvorhaben offenzulegen. Die Kriterien werden im weiteren Projektverlauf immer wieder hervorgeholt und zur Analyse der Fotos herangezogen. Hierüber sollen auch die Kriterien selbst - und damit Vorstellungen von (Nicht)Nachhaltigkeit - im Projektverlauf einer kritischen Revision unterzogen werden, bspw. wenn anfangs formulierte Vorstellungen von (Nicht-)Nachhaltigkeit den eigenen Forschungsergebnissen, konkreten lokalen Praktiken sowie den dialogisch in Erfahrung gebrachten (fachlich-politischen) Hintergründen nicht Stand halten.

Methodentraining: Photovoice. Photovoice ist eine niedrigschwellige partizipative Forschungsmethode, welche das Fotografieren als Datenerhebungsmethode mit Gruppendiskussionen innerhalb der Forscher*innengemeinschaft in ein produktives Verhältnis setzt und auf kollaborative Wissensproduktion abzielt. Sie ist unkompliziert einsetzbar, da das Aufnehmen und Teilen von Fotos im Web 2.0 wie selbstverständlich zur Erfahrungswelt von Jugendlichen gehört. Zugleich sind die unterschiedlichen Zugangsmöglichkeiten zu und Kompetenzen im Umgang mit Technologie innerhalb des Klassenverbandes zu berücksichtigen (bspw. bei der Zusammensetzung der Gruppen). Für die Umsetzung benennen Wihofszky et al. (2020: 89) drei Anliegen der Methode, die auch innerhalb der Forscher*innengemeinschaft herausgestellt und besprochen werden sollten: „Photovoice soll dazu beitragen, dass Menschen ihre Stärken und die Anliegen ihrer Gemeinschaft erfassen und reflektieren; die Methode soll einen kritischen Dialog und Wissen über persönliche und gemeinschaftliche
Fragen fördern, indem die Fotografien in Gruppen diskutiert werden; durch Photovoice sollen politische Entscheidungsträger*innen erreicht werden." Neben den Zielen der Methode sollten auch Grundlagen der Fotografie und die Bereiche Datenschutz und Forschungsethik thematisiert werden (Layh et al. 2020: 240). Fotografisch erfahrene Co-Forscher*innen können sich dabei als Expert*innen einbringen.

Forschungsvorhaben planen. Die Vorarbeiten münden in die Erarbeitung mehrerer Teilfragestellungen. In Kleingruppen werden die Feldphasen konkretisiert und auch das Zielpublikum bestimmt. Neben der Schulöffentlichkeit sollten dies im besten Fall auch lokale Akteur*innen und Entscheidungsträger*innen sein.

\section{Baustein 2: Forschungsprojekte verfolgen und reflektieren}

Es folgen mehrere Phasen der Aktion und Reflexion in zyklischer Abfolge, sodass „sukzessive ein neues, vertieftes Verständnis der Zusammenhänge erreicht und neue Handlungsansätze generiert" (Unger 2014: 60) werden. Unterrichtspraktisch bedingt die Nicht-Linearität von PAF ein kontinuierliches, dialogisches Problematisieren und Aushandeln der Vorhaben der Arbeitsgruppen und der gewonnenen Einsichten (vgl. Mountz et al. 2008). Sich im Unterricht auf diese Prozessualität von PAF-Vorhaben einzulassen bedeutet, Widerständiges und Fragwürdiges, Leerstellen und Umwege als Ausgangspunkte reflexiver Umlernprozesse wertzuschätzen und diesen Zeit und Raum zu geben (vgl. Pettig 2019). Prozessen des Umlernens geht ein krisenhaftes Ereignis voraus, welches den Moment markiert, in dem das gewohnte Denken nicht mehr reicht, eine bestimmte Situation einzuordnen und zu verstehen, hierüber Muster des Denkens sichtbar macht und nach neuer Antwort verlangt (vgl. Meyer-Drawe 1996, 2012). Wann dieser Prozess abgeschlossen ist, kann nur im jeweiligen Kontext bestimmt werden, z. B. wenn das Thema erschöpft ist oder die gemeinsame Zeit im Projekt vorüber ist.

Aktionen im Feld durchführen. Die Feldphasen dienen der Begegnung und Erkundung der Untersuchungsstandorte sowie dem Anfertigen von Fotos und ergänzenden Datensplittern (u. a. Notizen, Skizzen ...). Das Führen eines Forschungstagebuchs bietet sich an.

In den Dialog treten und die Vorhaben reflektieren. Die Diskussionsphasen dienen dem Austausch und der gemeinschaftlichen Aktualisierung des weiteren 
Vorgehens. Die strukturierte Reflexion der Fotografien ist wichtig, da die Aufnahmen nicht selbsterklärend sind und sich ihre Bedeutungen erst dialogisch preisgeben (Layh et al. 2020: 244). In Anlehnung an Unger (2014: 63) bietet sich für die gemeinschaftliche Analyse und Reflexion der Fotos ein mehrschrittiges Vorgehen an: Vorauswahl und Aufbereitung der Fotos durch die Arbeitsgruppen; Gruppierung der Fotos und Identifikation von Themen; Formulierung des übergeordneten Zusammenhangs. Hierüber kommen neben den Perspektiven und Intentionen der Fotograf*innen auch kollektive Erfahrungen und geteilte Sichtweisen zur Sprache. Die gemeinschaftliche Analyse und Reflexion der Fotos kann entsprechend dem in der Photovoice-Forschung etablierten „SHOWeD-Verfahren“ (Wang 1999: 188) entlang von fünf Fragen strukturiert werden, die hier adaptiert wurden:

1. Was ist auf dem Foto zu sehen?

2. Was sind die Hintergründe und Kontexte des Fotos?

3. Was hat das mit unserem Leben und Alltag zu tun?

4. Warum existiert diese nicht-nachhaltige Situation oder dieses Problem?/ Welche Lösung bietet die im Foto dargestellte Situation?

5. Was können wir tun, um das Problem anzugehen? / Was können wir tun, um diese Ideen zu unterstützen?

Die Dialogphase dient daraufhin der (Re-)Orientierung der jeweiligen Forschungsvorhaben. Es bietet sich an, an dieser Stelle auch den weiterführenden Austausch mit lokalen Akteur*innen (bspw. aus dem
Bereich alternativer Ökonomien, urbaner Gemeinschaftsgärten und Lebensmittelnetzwerke oder auch dem lokalen Quartiersentwicklungsbüro) zu suchen sowie auch themenbezogene Recherchen zu fachlichpolitischen Hintergründen und Zusammenhängen vorzunehmen.

\section{Baustein 3: Forschungsergebnisse zur Diskussion stellen und kritisch Stellung beziehen}

Im dritten Baustein werden zum einen die Forschungsvorhaben finalisiert, zum anderen die Ergebnisse in Vorbereitung auf die Präsentation kuratiert. Eine Vernissage lässt sich bspw. einfach mit einigen Stellwänden, anschaulich gestalteten Postern und prägnanten Kurzpräsentationen realisieren; auch $\mathrm{Vi-}$ deos, Broschüren oder Berichte in der Schulzeitung bieten sich an. Geeignete Rahmen für die Ausstellung können z. B. ein Tag der offenen Tür, ein Schul- oder Stadtteilfest, das Rathaus, oder auch Schulkonzerte sein. $\mathrm{Zu}$ diesen Veranstaltungen sollten auch eine breitere Öffentlichkeit und wünschenswerterweise auch lokale Entscheidungsträger*innen eingeladen werden, sodass die formulierten Anliegen, Einsichten und Lösungsvorschläge aus dem Forschungsprojekt auch möglichst lokale Impulse setzen und im besten Fall Prozesse der Transformation bei den CoForscher*innen selbst aber auch in der Community entfalten.

Ergebnisse kommunizieren. Zum Projektende werden die geplanten Maßnahmen umgesetzt (siehe Abb. 4).

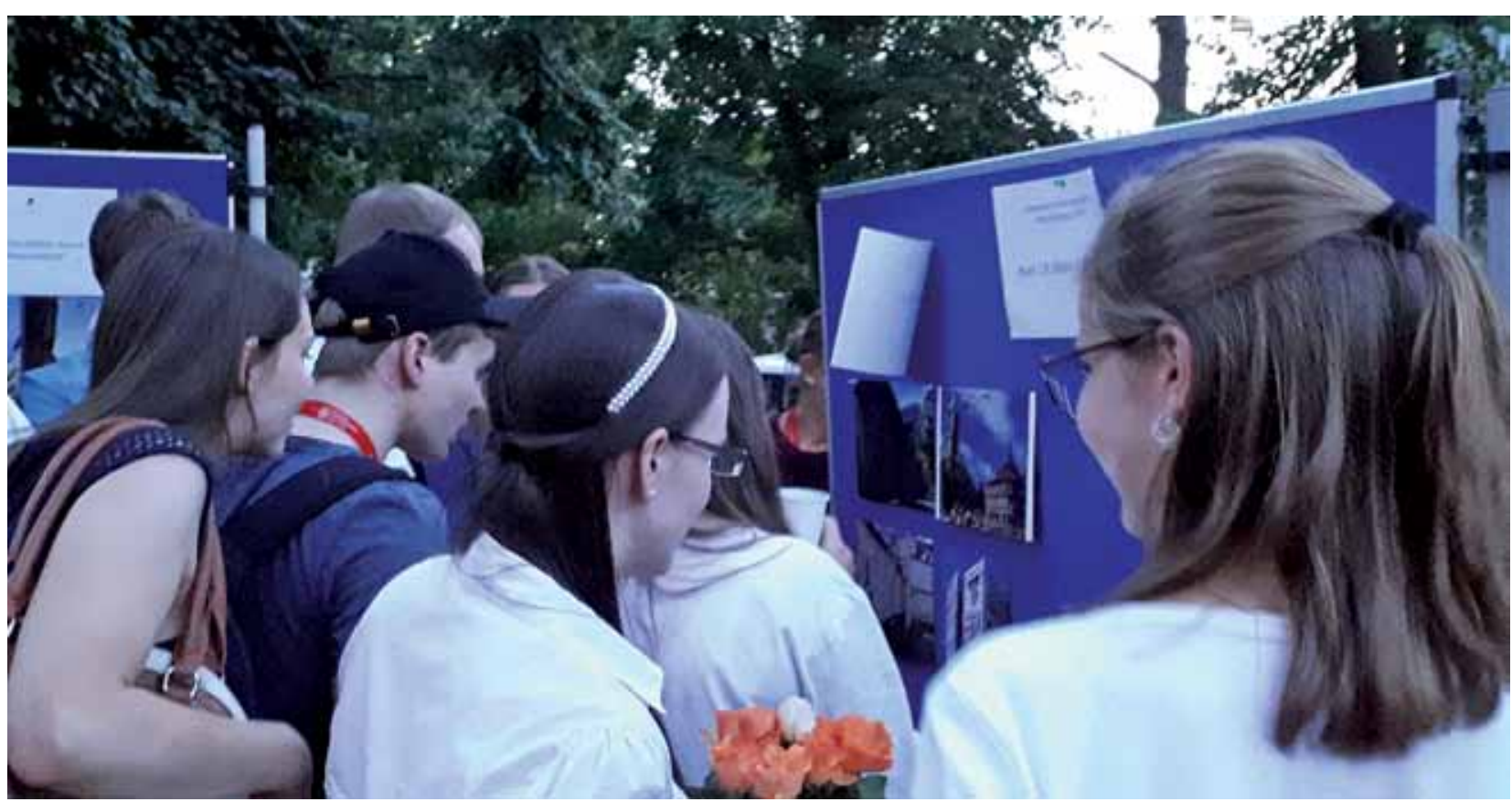

Abb. 4: Vernissage zu einem partizipativen Fotoprojekt mit Schüler*innen im Rahmen des Sommerkonzerts der Deutschen Schülerakademie 2018, Braunschweig. Die Co-Forscher*innen diskutierten ihre Ergebnisse mit anderen Teilnehmer*innen der Akademie und der geladenen Öffentlichkeit. (Foto: Pettig 2018) 
Debriefing in der Forscher*innengemeinschaft. In der letzten Phase wird die Forschung evaluiert. Im Anschluss an Unger (2014: 76) kann dies entlang einiger Impulsfragen gelingen, die hier erweitert wurden, um sich auch kritisch mit der Forschungsumgebung selbst und dem eigenen Lernprozess auseinanderzusetzen:

- Haben wir unsere Ziele im Projekt erreicht? Wenn nicht, warum nicht? Was hat zum Erreichen der Ziele beigetragen?

- Wie zufrieden seid Ihr mit Eurer Beteiligung im Projekt? Wo hättet Ihr Euch mehr Freiheiten gewünscht? Was waren Möglichkeiten und Grenzen des Projekts?

- Welche Auswirkungen hat das Projekt auf unseren Alltag, die Beteiligten oder die Untersuchungsgebiete?

- Was hat sich im Zuge des Projekts verändert? Was war unerwartet?

Einträge aus den Forschungstagebüchern können das Gespräch anreichern. Abschließend können über den Rahmen der Projektwoche hinausreichende Vereinbarungen getroffen werden, z. B. weitere Formen der Öffentlichkeitsarbeit, welche in Follow-Up-Aktivitäten realisiert werden können.

\section{Diskussion - Herausforderungen und Chancen von PAF in der geographischen Bildungspraxis}

PAF ist eine Art und Weise des kollaborativen Forschens bei der Co-Forscher*innen gemeinsam Veränderungsprozesse in ihrem Umfeld anzuregen versuchen. Dieser Forschungsstil bietet aus unserer Sicht einen wertvollen Denkrahmen für die Bildungspraxis, da er es ermöglicht, in Schule und Hochschule geographische Fragestellungen kritisch-reflexiv und aktionsorientiert zu bearbeiten. Dabei fordert der zirkuläre Charakter von PAF die beteiligten Akteur*innen auf mehreren Ebenen heraus, denn im Kern ist sie ein lehrreicher Prozess des Erarbeitens, Scheiterns und Überarbeitens. Der Reiz und die Bedeutung von PAF liegen genau darin, trotz aller Reibungen und Widerstände (immer wieder) neue Wege zu gehen und Widersprüche auszuhalten, um Wandel zu ermöglichen und gestaltbar zu machen. Die Leerstellen im Prozess nicht auszuklammern, sondern zum integralen Bestandteil des Settings zu machen, ist sowohl Anspruch als auch bildungstheoretischer Gewinn von PAF. Denn das Einlassen auf das Plötzliche, Unerwartete und Widerständige kann in seiner Bedeutung für Bildungsprozesse kaum hoch genug eingeschätzt werden.

Zugleich ist damit verbunden, dass PAF sich aufwendig und voraussetzungsvoll gestaltet und allen
Beteiligten viel abverlangt. So bedarf es auch eines gewissen Enthusiasmus sich auf die Aushandlungsprozesse einzulassen, um dialogisch die notwendigen Voraussetzungen für Partizipation und Engagement in Auseinandersetzung mit der Sache zu schaffen. Unserer Erfahrung nach nimmt die Bereitschaft aller Beteiligten sich hierauf einzulassen, im Laufe einer partizipativen Aktionsforschung tendenziell zu. Es sind vor allem die persönlichen Begegnungen und (Selbstwirksamkeits-)Erfahrungen im Zuge der konkret umgesetzten Aktionen, welche die Identifikation der Beteiligten mit dem Forschungsprozess erhöhen. Wie die Umsetzung der partizipativen Aktionsforschung gelingt und inwieweit deren transformative Potenziale ausgeschöpft werden, ist eng mit der individuellen Verbundenheit der Co-Forscher*innen mit dem jeweiligen Forschungsthema verbunden. Unsere Erfahrungen mit dem Einsatz non-verbaler Methoden, bspw. im Kontext von Hochschulseminaren, weisen darauf hin, dass diese überaus fruchtbar sind, um die Verbundenheit der Beteiligten mit den Themen zu fördern.

PAF ist also nicht nur ein Balanceakt zwischen Theorie und Praxis, Wissenschaft und Gesellschaft, schulischen und außerschulischen Kontexten, sondern auch zwischen lehrbuch- und erfahrungsbasiertem Wissen. Es gilt dabei die praktische Arbeit und die theoretische Reflexion in einen produktiven $\mathrm{Di}$ alog zu bringen und nicht einseitig aufzulösen. Für die konkrete Zusammenarbeit von Akteur*innen aus unterschiedlichen Kontexten (Wissenschaft, Schule, Praxis, Community) besteht die Herausforderung damit auch darin, respektvoll und aufmerksam zu sein und gleichzeitig den kritischen Blick nicht zu verlieren.

Unsere Erfahrungen in der Umsetzung von PAFProjekten zeigen, dass diese sowohl in der Schule als auch der Hochschule institutionalisierte Lehr-LernKontexte und -Formate herausfordern. Das Verhältnis von Lehrenden und Lernenden wird dabei einer kritischen Revision und in Zeiten multipler Krisen und unsicherer Zukünfte einer wertvollen Neuausrichtung unterzogen, in denen sozialer Wandel vorstellbar und machbar wird; oder in den Worten Paulo Freires (1971: 65): „Der Lehrer ist nicht länger bloß der, der lehrt, sondern einer, der selbst im Dialog mit den Schülern belehrt wird. [...] So werden sie miteinander für einen Prozess verantwortlich, in dem alle wachsen."

\section{Danksagung}

Die Autor*innen danken dem Open Access Publikationsfonds der Universität Graz für die Unterstützung. 


\section{Beitrag der Autor*innen}

F. P. hat den Beitrag strukturiert, die fachdidaktische Perspektive, den Unterrichtsbaustein und die Diskussion formuliert. L. V. hat die fachwissenschaftliche Perspektive formuliert und an der Diskussion mitgeschrieben. P. S. hat Erfahrungen aus Hochschulseminarkontexten formuliert und an der fachwissenschaftlichen Perspektive mitgeschrieben. S. H. hat an der fachwissenschaftlichen Perspektive und der Diskussion mitgeschrieben. M. N. hat Erfahrungen aus Hochschulseminarkontexten eingebracht. F. P. und L. V. haben den Beitrag auf Grundlage der anonymen Gutachten überarbeitet.

\section{Literatur}

Altrichter, H., P. Posch \& H. Spann (2018): Lehrerinnen und Lehrer erforschen ihren Unterricht. Klinkhardt (UTB), Bad Heilbrunn.

Arnstein, S. R. (1969): A ladder of participation. In: Journal of the American Planning Association 35(4). S. 216-224. DOI: https://doi.org/10.1080/01944366908977225

Bartholl, T. (2015): Territórios de resistência e movimentos sociais de base: uma investigação militante em favelas cariocas. Doktorarbeit. Universidade Federal Fluminense Rio de Janeiro, Brazilien. https://territoriosresistencia. wordpress.com/tese/ (25.09.2021).

Bergold, J. \& S. Thomas (2012): Partizipative Forschungsmethoden: Ein methodischer Ansatz in Bewegung. In: Forum: Qualitative Social Research 13(1). DOI: https:// doi.org/10.17169/fqs-13.1.1801

Castree, N. (2000): Professionalisation, Activism, and the University: Whither 'Critical Geography'? In: Environment and Planning A: Economy and Space 32(6). S. 955-970. DOI: https://doi.org/10.1068/a3263

Chambers, R. (1994): The Origins and Practice of Participatory Rural Appraisal. In: World Development 22(7). S. 953-969. DOI: http://dx.doi.org/10.1016/0305750X(94)90141-4

Chatterton, P., S. Hodkinson \& J. Pickerill (2010): Beyond scholar activism: making strategic interventions inside and outside the neoliberal university. In: ACME: An International Journal for Critical Geographies 9(2). S. $245-274$.

Cochran-Smith, M. \& S. L. Lytle (1993): Inside/Outside: Teacher Research and Knowledge. Teachers College Press, Columbia University, London (u. a.).

Counter Cartographies Collective, L. Mason-Deese \& C. Dalton (2012): Counter (Mapping) Actions - Mapping as Militant Research. In: ACME: An International Journal for Critical Geographies 11(3). S. 439-466.

Dammann, F. \& B. Michel (Hrsg.) (i. E.): Handbuch für Methoden kritischen Kartierens. UTB/transcript, Bielefeld.
Davidson, J. \& C. Milligan (2004): Embodying emotion sensing space: introducing emotional geographies. In: Social \& Cultural Geography 5(4). S. 523-532. DOI: https://doi.org/10.1080/1464936042000317677

Domann, V. \& S. Liebscher (2020): Forschende Lehre in einer polarisierten Gesellschaft - Perspektiven aus einer angewandten kritischen Geographie. In: GW-Unterricht 157. S. 56-64. DOI: https://doi.org/10.1553/gw-unterricht $157 \mathrm{~s} 56$

Eberth, A. \& V. Röll (2021): Reflexive Fotografie und Partizipation. In: Kogler, R. \& J. Wintzer (Hrsg.): Raum und Bild - Strategien visueller raumbezogener Forschung. Springer, Berlin/Heidelberg. S. 19-29.

Euler, P. (2014): Nachhaltigkeit und Bildung: Plädoyer für ein sachhaltiges Verstehen herrschender Widersprüche. In: Umweltdachverband (Hrsg.): Bildung für nachhaltige Entwicklung: Jahrbuch 2014. FORUM Umweltbildung, Wien. S. 167-173.

Evans, R., L. Holt \& T. Skelton (Hrsg.) (2017): Methodological Approaches. In: Geographies of Children and Young People Vol. 2. Springer, Singapore.

Fals Borda, O. (1978): El problema de cómo investigar la realidad para transformarla. Bogotá, Tercer Mundo.

Freire, P. (1971): Pädagogik der Unterdrückten: Bildung als Praxis der Freiheit. Rowohlt Taschenbuchverlag, Stuttgart.

Halder, S. \& P. Schweizer (2020): Von Aktivismus, Geographien und dem Dazwischen - Überlegungen anhand der Praxis von Kollektiv Orangotango. In: Standort 44(4). S. 255-261. DOI: https://doi.org/10.1007/s00548-02000681-8

Halder, S. (2018): Gemeinsam die Hände dreckig machen. transcript, Bielefeld.

Hart, R. A. (1992): Children's Participation: From Tokenism to Citizenship. UNICEF International Child Development Centre, Florence.

Hart, R. A. (2008): Stepping Back from "the ladder": Reflections on a model of participatory work with children. In: Reid, A., B. B. Jensen, J. Nikel \& V. Simovska (Eds.): Participation and Learning. Springer, Wiesbaden. S. 19-31.

Hartung, S., P. Wihofszky \& M. T. Wright (2020) (Hrsg.): Partizipative Forschung. Springer, Wiesbaden.

Herr, K. \& G. L. Anderson (2015): The Action Research Dissertation: A Guide for Students and Faculty. 2. Aufl. SAGE Publications, Thousand Oaks.

Kanwischer, D. \& T. Rhode-Jüchtern (2002): Aktionsforschung in der Schule am Beispiel einer Unterrichtseinheit zum Thema Unterentwicklung und Entwicklungszusammenarbeit. In: Kanwischer, D. \& T. Rhode-Jüchtern (Hrsg.): Qualitative Forschungsmethoden in der Geographiedidaktik. Bericht über einen HGD-Workshop in Jena, 21.-23. Juni 2001. HGD, Nürnberg. S. 131-152.

Katz, C. (1996): The expeditions of conjurers - Ethnography, power and pretense. In: Wolf, D. (Hg.): Feminist Dilemmas in Fieldwork. Westview, New York. S. $170-184$. 
Kehren, Y. \& C. Winkler (2019): Nachhaltigkeit als Bildungsprozess und Bildungsauftrag. In: Leal Filho, W. (Hg.): Aktuelle Ansätze zur Umsetzung der UN-Nachhaltigkeitsziele. Springer, Wiesbaden. S. 373-391.

Kerkhoff, L. van \& L. Lebel (2006): Linking Knowledge and Action for Sustainable Development. Annual Review of Environment and Resources 31. S. 445-477. DOI: https://doi.org/annurev.energy.31.102405.170850

Kindon, S. \& S. Elwood (2009): Introduction: More than Methods - Reflections on Participatory Action Research in Geographic Teaching, Learning and Research. In: Journal of Geography in Higher Education 33(1). S. 19-32. DOI: https://doi.org/10.1080/03098260802276474

Kindon, S., R. Pain \& M. Kesby (Hrsg.) (2007): Participatory Action Research Approaches and Methods: Connecting People, Participation and Place. Routledge, London (u. a.).

Kuge, J., M. Naumann, H. Nuissl \& S. Schipper (2020): Angewandte und Kritische Geographie. Gemeinsame Herausforderungen, gemeinsame Perspektiven? In: Standort 44(4). S. 219-225. DOI: https://doi.org/10.1007/ s00548-020-00662-x

Layh, S., A. Feldhorst, R. Althaus, M. Bradna \& P. Wihofszky (2020): Photovoice-Forschung mit Jugendlichen - ein Leitfaden zur Durchführung. In: Hartung, S., P. Wihofszky \& M. T. Wright (Hrsg.): Partizipative Forschung. Springer VS, Wiesbaden. S. 233-262. DOI: https://doi.org/10.1007/s00548-020-00662-x

Leder, S. (2015): Feldforschung, Dokumentenanalyse und Aktionsforschung: Ein geographiedidaktisches Forschungsdesign für den internationalen Kontext. In: Budke, A. \& M. Kuckuck (Hrsg.): Geographiedidaktische Forschungsmethoden. LIT, Münster. S. 269-291.

Lewin, K. (1946): Action research and minority problems. In: Journal of Social Issues 2(4). S. 34-46.

Meyer-Drawe, K. (1996): Vom anderen lernen: Phänomenologische Betrachtungen in der Pädagogik. In: Borrelli, M. \& J. Ruhloff (Hrsg.): Deutsche Gegenwartspädagogik Vol. 2. Schneider-Verlag, Hohengehren. S. 85-98.

Meyer-Drawe, K. (2012): Diskurse des Lernens. Wilhelm Fink, München.

Mießner, M. (2020): Angewandte Kritische Geographie am Beispiel der Wohnungsmarktforschung in Göttingen. In: Standort 44(4). S. 239-247. DOI: https://doi. org/10.1007/s00548-020-00665-8

Mordock, K. \& M. E. Krasny (2001): Participatory Action Research: A Theoretical and Practical Framework for EE. In: The Journal of Environmental Education 32(3). S. 15-20. DOI: https://doi.org/10.1080/00958960109599140

Mountz, A., E. B. Moore \& L. Brown (2008): Participatory action research as pedagogy: Boundaries in Syracuse. In: ACME: An International Journal for Critical Geographies 7(2). S. 214-238.

Pain, R. \& P. Francis (2003): Reflections on participatory research. In: Area 35(1). S. 46-54. DOI: https://doi. org/10.1111/1475-4762.00109
Pettig, F. (2019): Kartographische Streifzüge. Ein Baustein zur phänomenologischen Grundlegung der Geographiedidaktik. transcript, Bielefeld.

Pettig, F. (2021): Transformative Lernangebote kritischreflexiv gestalten. Fachdidaktische Orientierungen einer emanzipatorischen BNE. In: GW-Unterricht 162. S. 5-17. DOI: https://doi.org/10.1553/gw-unterricht162s5

Pichler, H., C. Hintermann \& D. Raithofer (2021): Identitätskonstruktion von Jugendlichen Trittsteine zur (De) Konstruktion von Identität(en) in der kritischen (geographischen) Medienbildung am Beispiel des Projektes MiDENTITY. In: GW-Unterricht 161. S. 19-34. DOI: https://doi.org/10.1553/gw-unterricht161s19

Pokraka,J.\&I.Gryl(2018):Kinder:Karten:Kommunikation - Spatial Citizenship zwischen Partizipation und Paternalismus. In: Kartographische Nachrichten 3. S. 140-146. DOI: https://doi.org/10.1007/BF03544557

Resch, K. \& M. Knapp (Hrsg.) (2020): Methoden Toolkit. Ein Vergleich praxisbezogener Lehransätze in der Hochschuldidaktik. Universität Wien, Wien. DOI: https:// doi.org/10.25365/phaidra.146

Rinschede, G. \& A. Siegmund (2020): Geographiedidaktik. 4. Aufl. Schöningh, Paderborn.

Rodó-de-Zárate, M. (2014): Developing geographies of intersectionality with Relief Maps: reflections from youth research in Manresa, Catalonia. In: Gender, Place \& Culture 21(8). S. 925-944. DOI: https://doi.org/10.1080/0 966369X.2013.817974

Routledge, P. (2017): Space Invaders: Radical Geographies of Protest. Pluto Press, London.

Routledge, P. (2009): Activist Geographies. In: Kitchin, R. $\& \mathrm{~N}$. Thrift (Hrsg.): International Encyclopedia of $\mathrm{Hu}-$ man Geography. Elsevier, Amsterdam/Oxford. S. 7-14.

Scheidel, A., D. Del Bene, J. Liu, G. Navas, S. Mingorría, F. Demaria, S. Avila, B. Roy, I. Ertör, L. Temper \& J. Martínez-Alier (2020): Environmental conflicts and defenders: A global overview. In: Global Environmental Change 63. S. 102104. DOI: https://doi.org/10.1016/j. gloenvcha.2020.102104

Schulze, U., D. Kanwischer \& O. Wolff (2018): ServiceLearning and Pre-Service Teacher Education: A Case Example from Geography. In: Zeitschrift für Hochschulentwicklung 13(2). S. 113-126. DOI: https://doi. org/10.3217/zfhe-13-02/07

Segebart, D. (2007): Partizipatives Monitoring als Instrument zur Umsetzung von Good Local Governance: eine Aktionsforschung im östlichen Amazonien/Brasilien. Selbstverlag Geographisches Institut der Universität Tübingen, Tübingen.

Singer, K. (2019): Confluencing Worlds. Skizzen zur Kolonialität von Kindheit, Natur und Forschung im Callejón de Huaylas, Peru. Dissertationsschrift. Institut für Geographie der Universität Hamburg, Hamburg.

Singer-Brodowski, M. (2016): Transformatives Lernen als neue Theorie-Perspektive in der BNE. In: Umweltdachverband (Hrsg.): Jahrbuch Bildung für nachhaltige Ent- 
wicklung - Im Wandel. Forum Umweltbildung, Wien. S. $130-139$.

Steinke, I. (2010): Gütekriterien qualitativer Forschung. In: Flick, U., E. von Kardorff \& I. Steinke (Hrsg.): Qualitative Forschung - Ein Handbuch. Rowohlt, Reinbek. S. 319-331.

Sousa Santos, B. de (2018): The End of the Cognitive Empire: The Coming of Age of Epistemologies of the South. Duke University Press, Durham/London.

Unger, H. von (2014): Partizipative Forschung. Springer VS, Wiesbaden. DOI: https://doi.org/10.1007/978-3658-01290-8

Vare, P. \& W. Scott (2007): Learning for a Change: Exploring the Relationship Between Education and Sustainable Development. In: Journal of Education for Sustainable Development 1(2). S. 191-198. DOI: https://doi. org/10.1177/097340820700100209\#

Wang, C. C. \& M. A. Burris (1994): Empowerment through photo novella: portraits of participation. In: Health Education \& Behavior 21(2). S. 171-186. DOI: https://doi.org/10.1177/109019819402100204
Wang, C. C. \& M. A. Burris (1997): Photovoice: concept, methodology, and use for participatory needs assessment. In: Health Education \& Behavior 24(3). S. 369-387. DOI: https://doi.org/10.1177/109019819702400309

Wang, C. C. (1999): A Participatory Action Research Strategy Applied to Women's Health. In: Journal of Women's Health 8(2). S. 187-192. DOI: https://doi.org/10.1089/ jwh.1999.8.185

Wihofszky, P., S. Hartung, T. Allweiss, M. Bradna, S. Brandes, D. Gebhardt \& S. Layh (2020): Photovoice als partizipative Methode: Wirkungen auf individueller, gemeinschaftlicher und gesellschaftlicher Ebene. In: Hartung, S., P. Wihofszky \& M. T. Wright (Hrsg.): Partizipative Forschung. Springer VS, Wiesbaden. S. 85-141. DOI: https://doi.org/10.1007/978-3-658-30361-7_4

Wöhrer, V., D. Arztmann, T. Wintersteller, D. Harrasser \& K. Schneider (2017): Partizipative Aktionsforschung mit Kindern und Jugendlichen. Springer VS, Wiesbaden.

Zibechi, R. (2011): Territorien des Widerstands: Eine politische Kartografie der urbanen Peripherien Lateinamerikas. Assoziation A, Berlin. 